\title{
The electrokinetic properties of latex particles: comparison of electrophoresis and dielectrophoresis
}

\author{
Irina Ermolina, Hywel Morgan* \\ School of Electronics and Computer Science, The University of Southampton, Highfield, Southampton SO17 1BJ, UK
}

Received 13 August 2004; accepted 3 November 2004

Available online 22 December 2004

\begin{abstract}
A comprehensive study of the AC and DC electrokinetic properties of submicrometre latex particles as a function of particle size and suspending medium conductivity and viscosity is presented. Electrophoretic mobility and dielectrophoretic cross-over results were measured for particle diameters ranging from 44 to $2000 \mathrm{~nm}$. The zeta potentials of the particles were calculated from the electrophoretic mobility data for different suspending medium conductivities, using various models, with and without the inclusion of surface conduction. The dielectrophoretic data was analysed to derive values for the Stern layer conductance and zeta potentials.
\end{abstract}

(C) 2004 Elsevier Inc. All rights reserved.

Keywords: Latex spheres; Surface conductance; Stern layer; Diffuse layer; Nanoparticles; Electrokinetic properties

\section{Introduction}

Both the AC and DC electrical properties of micrometre and submicrometre latex particles have been investigated by many research groups, using techniques such as electrophoresis, electrical conductivity measurements, electroacoustics and dielectric spectroscopy; see, for example, [1-19].

It has been observed that the electrophoretic behaviour of latex particles cannot be completely explained using the conventional Gouy-Chapman model of the double layer. Plots of zeta potential against electrolyte concentration always showed peaks in the region of 1 to $10 \mathrm{mM}$, whereas a monotonic decrease with electrolyte concentration would be expected (e.g., $[6,7,20])$. The behaviour of the particles can be better modelled by introducing the concept of a surface conductance, where charge is considered to move in a thin layer outside the shear or slip plane [21,22]. Dukhin and Deryaguin [1] introduced the concept of "anomalous" surface conductance, where it was assumed that charge move-

\footnotetext{
* Corresponding author.

E-mail address: hm@ecs.soton.ac.uk (H. Morgan).
}

ment occurred between the shear plane and the particle surface. In this model the shear plane was located some way into the diffuse layers so that the ions contributing to the surface conductance effectively move in the diffuse layer with a high mobility. Other authors $[7,8,12]$ introduced the concept of a surface conduction arising from charge movement in the region behind the slip plane. This conductance contains two components, one due to convection of ions due to electric field-driven movement of the liquid (electroosmosis) and another due to the movement of the ions directly from the action of the electric field on the charge, the conduction current.

As discussed by Hunter [23], the stagnant layer charge could be behind or in front of the shear plane depending on the relative amounts of charge in the diffuse layer and the surface charge density. Conduction behind the slip plane leads to a higher value of the zeta potential at low conductivities.

Measurements of the dielectric properties of latex particles have also shown that the inclusion of a surface conduction behind the shear plane is necessary to model the data [5, $7,21]$. The concept of a general surface conductance within dielectric theory has been around for 70 years. Bikerman 
[23] obtained an expression for the dielectric constant of a suspension or cylindrical rods with surface conductance. Miles and Robertson [24] and O'Konski [25] derived expressions for the dipole moment of a particle with arbitrary surface conductance. However, these models only partly account for the data. For example, Rasmusson et al. [16] failed to model their dielectric data by including surface conduction in the Stern layer; they only succeeded in matching the relaxation time.

Recent data concerning the dielectrophoretic properties of latex particles shows that the polarisation of the particles is dominated by surface conductance [26-28]. O'Konski's theory of constant surface conductance can explain the $\mathrm{AC}$ electrokinetic behaviour of micrometre-sized latex particles [28]. However, it does not adequately model the behaviour of submicrometre latex particles [26,27]. Again, the discrepancies have been attributed to the dominance of charge movement in the stagnant (or Stern) layer, beyond the slip plane rather than in the diffuse part of the double layer [27]. Neglecting contributions from the diffusion layer, the total surface conductance of the particle can be modelled as the sum of the Stern layer and diffuse layer conductance [7]. Using this approach, it has been possible to fit dielectrophoretic cross-over data for nanometre-scale latex particles measured at different suspending medium conductivities $[27,29,30]$.

In this paper we present a comprehensive study of the electrokinetic properties of submicrometre latex particles as a function of particle size and suspending medium conductivity and viscosity. Both electrophoretic mobility and dielectrophoretic cross-over results are presented for particle diameters ranging from 44 to $2000 \mathrm{~nm}$. The zeta potentials of the particles were calculated from the electrophoretic mobility data for different suspending media conductivities, with and without the inclusion of surface conduction. The dielectrophoretic data was analysed to derive values for the Stern layer conductance and zeta potentials.

The mobility of the ions that give rise to surface conductance behind the shear plane is unknown, but experiments have shown that it is close to that of the ions in the bulk [7, $8,21,30,31]$. Therefore, in order to investigate the role of the Stern layer in determining the DEP properties of particles, we also measured the dielectrophoretic cross-over data of latex particles in suspending media of different viscosities. An empirical model that describes the Stern-layer mobility for different viscosities is presented.

\section{Theory}

\subsection{AC electrokinetics and dielectrophoresis}

The dielectrophoretic (DEP) force acting on a polarisable particle in nonuniform field is proportional to real part of the
Clausius-Mossotti factor, given by [32-34]

$\operatorname{Re}\left\{f_{\mathrm{CM}}\right\}=\operatorname{Re}\left\{\frac{\varepsilon_{\mathrm{p}}^{*}-\varepsilon_{\mathrm{m}}^{*}}{\varepsilon_{\mathrm{p}}^{*}+2 \varepsilon_{\mathrm{m}}^{*}}\right\}$,

where $\varepsilon_{\mathrm{p}}^{*}$ and $\varepsilon_{\mathrm{m}}^{*}$ are the complex dielectric permittivities of the particle and medium, respectively, defined by $\varepsilon^{*}=$ $\varepsilon-j \sigma / \omega$, where $j^{2}=-1, \varepsilon$ is the dielectric constant and $\sigma$ the conductivity.

The real part of the Clausius-Mossotti factor is bounded by the limits $1<\operatorname{Re}\left\{f_{\mathrm{CM}}\right\}<-1 / 2$ and, as shown by Eq. (1), varies with the frequency of the applied field and dielectric parameters of particle and medium. Depending on the sign of $\operatorname{Re}\left\{f_{\mathrm{CM}}\right\}$, positive or negative DEP occurs, resulting in motion of particles towards high or low field strengths. Experimentally the particles are observed to collect at electrode edges or be repelled away from them, respectively. The cross-over frequency $\left(f_{X}\right)$ is defined as the value of the applied frequency which results in the cessation of particle motion. Therefore, measurement of the cross-over frequency can be used to characterise the dielectric properties of single particles. For any given suspending medium conductivity and permittivity, the cross-over frequency for a solid homogeneous spherical particle is uniquely defined by

$f_{X}=\frac{1}{2 \pi \varepsilon_{0}}\left(\frac{\sigma_{\mathrm{p}}^{2}+\sigma_{\mathrm{p}} \sigma_{\mathrm{m}}-2 \sigma_{\mathrm{m}}^{2}}{\varepsilon_{\mathrm{p}}^{2}+\varepsilon_{\mathrm{p}} \varepsilon_{\mathrm{m}}-2 \varepsilon_{\mathrm{m}}^{2}}\right)^{1 / 2}$.

At the cross-over frequency, the polarisability of the particle and fluid are the same and the particle ceases to experience any DEP force. Therefore, measurement of the cross-over frequency is not complicated by movement of the particle relative to the fluid; however, fluid movement can occur due to electrohydrodynamic effects such as AC electroosmosis [34,35]. At low frequencies, the DEP behaviour of submicrometre particles is dominated by the conductivity component $\left(\sigma_{\mathrm{p}}\right)$ of the polarisability. The bulk conductivity of latex beads is negligible and the particle conductivity is dominated by the movement of charges associated with the particle surface and the electrical double layer.

As shown in the work of O'Konski [25], the conductivity of a solid homogeneous spherical particle (such as a latex sphere) is given by the sum of the bulk conductivity, $\sigma_{\mathrm{b}} \cong 0$, and a surface conductivity given by

$\sigma_{\mathrm{p}}=\sigma_{\mathrm{b}}+\frac{2 K_{\mathrm{s}}}{a}$,

where $K_{\mathrm{S}}$ is a general surface conductance (typically $1 \mathrm{nS}$ for latex particles), and $a$ the particle radius.

As described in the Introduction, the inclusion of a surface conductance into the general expression for the dipole has been used to model the properties of latex particles larger than $1 \mu \mathrm{m}$, but fails to do so for smaller particles. Following on from the work of Dukhin and Lyklema amongst others [1,7], the surface conductance of the particle can be divided into two distinct components, one due to charge movement in the Stern or stagnant layer and another due to charge 
movement in the diffuse part of the double layer. Equation (3) then becomes [27]

$\sigma_{\mathrm{p}}=\sigma_{\mathrm{b}}+2 \frac{K_{\text {Stern }}}{a}+2 \frac{K_{\text {Diff }}}{a}$,

where $K_{\text {Stern }}$ and $K_{\text {Diff }}$ are the Stern layer conductance and the diffuse layer conductance, respectively. The Stern layer conductance is given by

$K_{\text {Stern }}=\rho_{\mathrm{q}, \text { Stern }} \mu_{\text {Stern }}$,

where $\rho_{\mathrm{q}, \text { Stern }}$ and $\mu_{\text {Stern }}$ are the equivalent surface charge density and ion mobility in the Stern layer.

The diffuse layer conductance consists of two components, one from electroosmosis caused by fluid movement beyond the shear plane $K_{\text {Diff,eo }}$, and the other a conduction current caused by the movement of charge with respect to the liquid $K_{\text {Diff,m }}$ [31]. The diffuse layer conductance is [7, 31]

$$
\begin{aligned}
K_{\text {Diff }} & =K_{\text {Diff, } \mathrm{m}}+K_{\text {Diff,eo }} \\
& =\frac{4 F^{2} c z^{2} D\left(1+3 m / z^{2}\right)}{R T \kappa}\left(\cosh \left[\frac{z q \zeta}{2 k T}\right]-1\right),
\end{aligned}
$$

where $\zeta$ is the zeta potential (the potential at the surface of shear), $D$ the diffusion coefficient of the excess free charge in the diffuse layer, $z$ the valence of the counterion, $F$ the Faraday constant, $R$ the gas constant, $k$ Boltzmann's constant, $q$ the charge on the electron and $T$ the temperature. The inverse Debye length is given by $\kappa=$ $\sqrt{\left(2 c z F^{2}\right) /\left(\varepsilon_{\mathrm{m}} R T\right)}$, where $c$ is electrolyte concentration $\left(\mathrm{mol} \mathrm{m}^{-3}\right)$. In Eq. (6) the dimensionless parameter $m$ describes the contribution of the electroosmotic ion flux to the diffuse layer surface conductance,

$m=\left(\frac{R T}{F}\right)^{2} \frac{2 \varepsilon_{\mathrm{m}}}{3 \eta D}$,

where $\eta$ is the viscosity of suspending solution.

In this work, the surface conductivity of the latex particles was determined from the DEP cross-over data using the above set of equations. Data was fitted to obtain both the Stern layer conductance and the zeta potential of the particles (via Eq. (6)).

\subsection{DC electrokinetics: electrophoresis}

For a particle with a thin double layer $(k a \gg 1)$, and ignoring the influence of surface conductance, the zeta potential $\zeta$ can be calculated from the electrophoretic mobility using the well-known Helmholtz-Smoluchowski equation:

$\mu_{\mathrm{El}}=\frac{\varepsilon_{\mathrm{m}} \zeta}{\eta}$.

The other limiting case, for $k a \ll 1$, is given by Hückel's formula $[20,34]$ :

$\mu_{\mathrm{El}}=\frac{2 \varepsilon_{\mathrm{m}} \zeta}{3 \eta}$.
Henry [34] determined an expression for arbitrary values of $k a$ (at low $\zeta$ potential), while Ohshima [20,36] produced the following simplified analytical expression:

$\mu_{\mathrm{El}}=\frac{2}{3} \frac{\varepsilon \zeta}{\eta}\left(1+\frac{1}{2\left[1+2.5 /\left\{\kappa a\left(1+2 e^{-\kappa a}\right)\right\}\right]^{3}}\right)$.

Dukhin and co-workers [1] presented an analytical model for the polarisation of the double layer, which included surface conduction and which is applicable for high values of $k a(>10)$. This relates the electrophoretic mobility to the zeta potential in the same way as the HelmholtzSmoluchowski expression but with a modification due to the surface conductance. Defining the nondimensional zeta potential in the usual way, $\bar{\zeta}=\zeta q /(k T)$, the expression derived by Dukhin is [1]

$$
\begin{aligned}
\frac{\eta q}{\varepsilon k T} \mu_{\mathrm{El}} & \\
=\bar{\zeta}-4( & \bar{\zeta}\left(1+3 m / z^{2}\right) \sinh ^{2}\left(\frac{z \bar{\zeta}}{4}\right) \\
& \left.+\left[\frac{2}{z} \sinh \left(\frac{z \bar{\zeta}}{2}\right)-3 m \bar{\zeta}\right] \ln \cosh \left(\frac{z \bar{\zeta}}{4}\right)\right) \\
/(\kappa a & +\left[8\left(1+3 m / z^{2}\right) \sinh ^{2}\left(\frac{z \bar{\zeta}}{4}\right)\right. \\
& \left.\left.-\left(24 m / z^{2}\right) \ln \cosh \left(\frac{z \bar{\zeta}}{4}\right)\right]\right) .
\end{aligned}
$$

A similar expression was derived by O'Brien and Hunter [37] and is given by

$$
\frac{\eta q}{\varepsilon k T} \mu_{\mathrm{El}}=\bar{\zeta}-\frac{\bar{\zeta}-\frac{2 \ln 2}{z}[1-\exp (-z \bar{\zeta})]}{1+\left[\kappa a \exp \left(-\frac{z \bar{\zeta}}{2}\right)\right] / 2\left(1+3 m / z^{2}\right)} .
$$

Shubin et al. [38] derived an expression which included the effect of surface conduction through the introduction of what is now referred to as the Dukhin number, Du. This parameter explicitly reflects the contribution made to the polarisation by surface conductance, given by $K_{\mathrm{s}} /\left(a \sigma_{\mathrm{m}}\right)$. This equation for the mobility then becomes (for a derivation see the appendix of Ref. [38])

$$
\frac{\eta q}{\varepsilon k T} \mu_{\mathrm{El}}=\bar{\zeta}+\frac{2 \mathrm{Du}}{1+2 \mathrm{Du}}\left\{\frac{2 \ln 2}{z}-2 \ln \left[1+\exp \left(\frac{z \bar{\zeta}}{2}\right)\right]\right\}
$$

From Eq. (6) the Dukhin number can be written as

$\mathrm{Du}=\frac{1}{\kappa a}\left(1+\frac{3 m}{z}+\Theta\right)[\exp (\bar{\zeta} / 2)-1]$.

In this expression the additional parameter $\Theta$ reflects the contribution to the total surface conduction made by conduction behind the shear plane [7,31], given by the ratio of the Stern layer conduction to the conduction current in the diffuse layer, in the absence of electroosmosis, i.e., 
Table 1

Concentration of Ficoll, together with corresponding suspending medium viscosities and conductivities of the solutions used for dielectrophoretic measurements

\begin{tabular}{|c|c|c|c|c|c|c|c|c|c|c|}
\hline & \multicolumn{10}{|c|}{ Ficoll (\%) } \\
\hline & 0 & 0.5 & 1 & 2 & 4 & 6 & 8 & 10 & 12 & 14 \\
\hline Viscosity $\left(\mathrm{kg} \mathrm{m}^{-1} \mathrm{~s}^{-1}\right) \times 10^{-3}$ & 1 & 1.1 & 1.2 & 1.45 & 2 & 2.8 & 3.9 & 5.4 & 7 & 9.2 \\
\hline Conductivity $\left(\mathrm{mS} \mathrm{m}^{-1}\right)$ & 0.1 & 0.16 & 0.22 & 0.34 & 0.59 & 0.83 & 1.07 & 1.31 & 1.55 & 1.8 \\
\hline
\end{tabular}

$\Theta=K_{\text {Stern }} / K_{\text {Diff,m }}$. For small values of the Dukhin number (i.e., Du «1), Eqs. (11)-(14) reduce to the HelmholtzSmoluchowski limit given by Eq. (8). In Eq. (13) the second term gives the degree of retardation to the electrophoretic mobility from the polarisation of the double layer. Note that these expressions hold true only for the thin double layer limit, i.e., $\kappa a>10$.

\section{Material and methods}

\subsection{Latex spheres}

Carboxy-functionalised fluorescent latex spheres ranging from 44 to $2000 \mathrm{~nm}$ in diameter were obtained from Molecular Probes (Oregon, USA). Stock solutions as supplied contained $2 \% \mathrm{w} / \mathrm{v}$ beads suspended in pure water, with a conductivity of less than $15 \mathrm{mS} / \mathrm{m}$. For the experiments, separate suspensions of beads were prepared by diluting small samples of stock solutions by a factor of 1000 into $\mathrm{KCl}$ electrolyte at a range of conductivities from 0.05 to $150 \mathrm{mS} / \mathrm{m}$. The samples were used for both electrophoretic mobility and DEP cross-over measurements without further modification. All suspensions were vortexed for $1 \mathrm{~min}$ and sonicated for $1 \mathrm{~min}$ prior to use. Beads were not centrifuged as this has been shown to alter their properties [20]. The DEP measurements were performed at room temperature (not controlled, but approximately $23^{\circ} \mathrm{C}$ ), whilst the electrophoretic mobility measurements were performed in a temperaturecontrolled sample cell at $25^{\circ} \mathrm{C}$. All samples were allowed to reach room temperature before measurements were taken.

Measurements of the dielectrophoretic cross-over frequency at different suspending medium viscosities were made using $\mathrm{KCl}$ containing Ficoll at different concentrations. The procedure was as follows: a stock solution of Ficoll at $14 \% \mathrm{w} / \mathrm{v}$ was prepared in $0.1 \mathrm{mM} \mathrm{KCl}$. This solution was dialysed against $\mathrm{RO}$ water for $30 \mathrm{~h}$ to remove the salt present in the stock Ficoll. The final conductivity of the stock solution was $1.8 \mathrm{mS} / \mathrm{m}$. Solutions covering a range of viscosities were prepared by serial dilution of the stock with $\mathrm{RO}$ water. The absolute viscosity of the samples was calculated with reference to a published calibration plot [39]. The values of viscosity and conductivity of these samples are presented in Table 1.

The permittivity of the Ficoll was measured using a HP 4192A impedance analyser at a fixed frequency of $5 \mathrm{MHz}$ with a microfabricated sample cell. At the maximum con- centration $(14 \% \mathrm{w} / \mathrm{v})$ the change in permittivity of the suspending medium was minimal, decreasing from 78 (pure water) to $76.5 \pm 0.5$. In view of this result, the data was analysed assuming that the Ficoll did not alter the suspending medium permittivity.

\subsection{Electrophoretic mobility measurements}

The electrophoretic mobility, $\mu_{\mathrm{El}}$, of the particles was measured with a commercial zeta potential analyser (Coulter DELSA 440 Series, Beckman Coulter, UK). The electrophoretic velocity of the particles was measured at the stationary layer of the fluid, which was at a point in the sample cell predetermined by the manufacturer of the sample cell.

\subsection{Dielectrophoretic measurements}

The DEP cross-over frequency was measured by observing particle motion at the edge of electrodes with castellated features. These devices were fabricated on glass microscope slides using direct-write electron-beam lithography. Three different electrode sizes were used with characteristic feature sizes of 5, 7 and $10 \mu \mathrm{m}$ (along all faces). They were made from a layer of $100 \mathrm{~nm}$ Au sandwiched between two $10 \mathrm{~nm}$ thick layers of Ti. Electrodes were powered using a signal generator providing $5 \mathrm{~V}$ peak-to-peak sinusoidal signals over the frequency range of $1 \mathrm{~Hz}$ to $20 \mathrm{MHz}$. Particles were observed using an epifluorescence microscope. For the measurements, approximately $30 \mu \mathrm{l}$ of particle suspension at a concentration of approximately $\left(2 \times 10^{-3}\right) \% \mathrm{w} / \mathrm{v}$ was pipetted into the electrode chamber and the assembly sealed with a coverslip.

\section{Results and discussion}

The cross-over data for latex particles ranging from 44 to $1900 \mathrm{~nm}$ in diameter are shown in Fig. 1. The data are plotted for beads suspended in $\mathrm{KCl}$ solutions of different molarities, with corresponding conductivities ranging from $10^{-4}$ to $10^{-1} \mathrm{~S} / \mathrm{m}$. Each data point is the average of 3 repeated measurements, using 3 different electrode sizes, measured in duplicate (18 total measurements). The error bars show the standard deviation. The data conforms to the general trend observed for latex particles, with a single cross-over from positive to negative DEP occurring in the $\mathrm{MHz}$ region [27]. At high suspending medium conductivities the particles experience only negative DEP. In the high conductivity region, 
Table 2

Stern layer conductance $K_{\text {Stern }}$ and $\zeta$-potential determined from fitting the dielectrophoretic data (cross-over frequency) shown in Fig. 1 with Eqs. (2) and (4)-(7)

\begin{tabular}{lllllll}
\hline & \multicolumn{2}{l}{ Particle diameter $(\mathrm{nm})$} & & & \\
\cline { 2 - 7 } & 44 & 110 & 216 & 460 & 557 & 996 \\
\hline$K_{\text {Stern }}(\mathrm{nS})$ & $\sim 0.75$ & 0.92 & 0.9 & 1.15 & 1.05 & 1.12 \\
$\zeta(\mathrm{mV})$ DEP & $\sim 0.075$ & 0.067 & 0.05 & 0.06 & 0.045 & 0.045 \\
\hline
\end{tabular}

The data for the $44 \mathrm{~nm}$ diameter particles is only approximate due to the difficulty in measuring these particles. Because Eqs. (2) and (4)-(7) imply the thin double layer limit, their applicability to the smallest particles at the lowest conductivities is probably not justified.

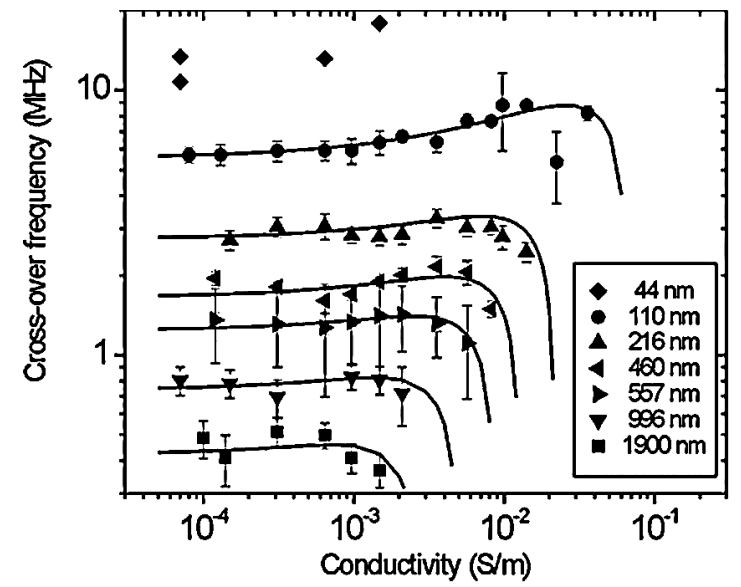

Fig. 1. Cross-over frequency for different sizes of beads plotted as a function of suspending medium conductivity. Solid lines are the best fit to the model described by Eqs. (2) and (4)-(7). The fitting data are summarised in Table 2 .

the measurements are also compounded by electrohydrodynamic effects [34,35].

Fig. 1 shows that the cross-over frequency for particles larger than $1 \mu \mathrm{m}$ is almost independent of the suspending medium conductivity. For smaller particles, the cross-over frequency increases with increasing suspending medium conductivity, as noted previously [26,27,29]. Also shown in this figure is the best fit to each data set obtained using Eqs. (2)-(7). The fits were obtained by varying only two parameters: the $\zeta$-potential and $K_{\text {Stern }}$. The mobility of the excess ion in the diffusion layer was set equal to that of the bulk. Typical values of Stern layer conductance were in the range of 1 to $2 \mathrm{nS}$. The diffuse layer conductance (as given by Eq. (6)) was typically one to two orders of magnitude lower than this, so that for suspending medium conductivities up to $100 \mathrm{mS} / \mathrm{m}$, conductance in the Stern layer dominates the behaviour of the particles. The results of this fitting are presented in Table 2 .

The electrophoretic mobility of all the beads was measured in a range of $\mathrm{KCl}$ solutions with conductivities from $10^{-4}$ to $10^{-1} \mathrm{~S} / \mathrm{m}$. Fig. 2 shows the data for four representative samples of beads. For the three largest particles shown in the figure, the mobility reaches its maximum at a molarity in the region of $10^{-2} \mathrm{M}$. For the smallest particle size (44 nm) no maximum was observed in the mobility data over the range of molarities measured. Fig. 3 shows a graph $(\log -\log )$ of the conductivity (or molarity), at which the peaks in the mobility values occur, plotted against particle diameter. This figure illustrates how the mobility maximum moves to lower ionic strength as the particle radius increases. This increase in mobility of particles with molarity has been reported previously (e.g., [1-7]). For example, Vorwerg et al. [17] noted that for 520 and $254 \mathrm{~nm}$ diameter particles, the mobility reached its maximum at approximately $10 \mathrm{mM} \mathrm{KCl}$, whilst the data for $31 \mathrm{~nm}$ diameter particles showed no such trend. Similar results were reported by Bensley and Hunter [4]. As noted by other workers, the conductivity at which the maximum in the electrophoretic mobility occurs depends on the particle size. This is due to the fact that the Dukhin number $\mathrm{Du}$ is given by $K s /\left(a \sigma_{\mathrm{m}}\right)$; therefore a lower $\sigma_{\mathrm{m}}$ is required to offset the higher value of $a$ [40].

The $\zeta$-potential for the particles was calculated from the electrophoretic mobility data using different models, and this data is shown in Fig. 4 for the same particle diameters as shown in Fig. 2. The analytical expression of Ohshima, given by Eq. (10) (which ignores surface conduction), was used to calculate the zeta potential for arbitrary $\kappa a$. This fit is shown in the figures for the four bead sizes, and shows that the zeta potential mirrors the variation in electrophoretic mobility seen in Fig. 2, with a maximum in the values for the larger diameter particles occurring in the conductivities of $10^{-2}$ to $10^{-1} \mathrm{~S} / \mathrm{m}$.

The zeta potential was also calculated using the three different models given by Eqs. (11)-(14) which include the effect of surface conduction beyond the shear plane but without Stern layer conduction, i.e., setting $\Theta=0$ in Eq. (13). Using the model of Shubin et al., Eq. (13), we also calculated the zeta potential with $\Theta$ set to a value given by the Stern layer conductance determined from the best fit to the DEP data (Table 2). The resulting set of curves is shown in Fig. 4. For the largest particle (1871 nm diameter) all three models produce the same trend, with the zeta potential reducing to approximately $50 \mathrm{mV}$ at low suspending medium conductivities.

As found by other workers, the inclusion of a finite Stern layer conductance produces a larger zeta potential, typically $100 \mathrm{mV}$ for the bigger particles. Note that the models presented above do not apply for thick double layer, so the values below $\kappa a \approx 10$ should be ignored. The $996 \mathrm{~nm}$ beads show similar behaviour, providing the last data points at $\kappa a<10$ are discounted. Again, the limiting low molarity zeta potential is of the order of $80 \mathrm{mV}$ when the Stern layer conductance is included. The data for the smaller particles 

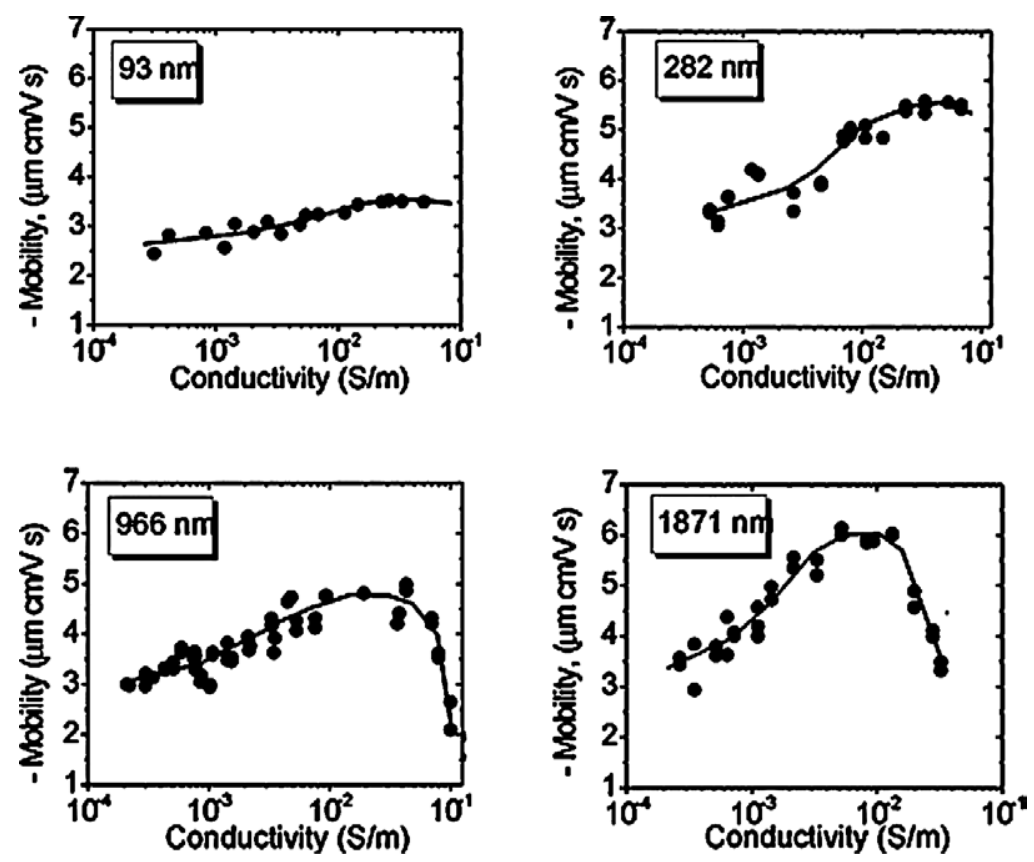

Fig. 2. Electrophoretic mobility data for $93,282,966$ and $1871 \mathrm{~nm}$ latex beads plotted against suspending medium conductivity.

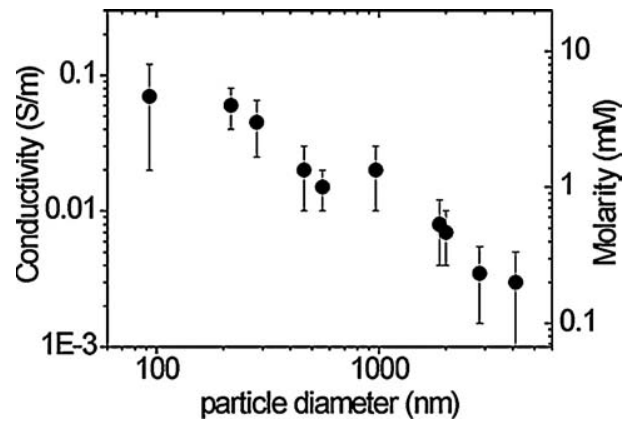

Fig. 3. The conductivity (and equivalent molarity of $\mathrm{KCl}$ ) at which the electrophoretic mobility of the latex particles reaches its maximum value, plotted as a function of particle diameter.

is more difficult to interpret because most of the conductivities lie below $\kappa a<10$. There is also a greater discrepancy between the different models. At high molarities, the zeta potential is approximately $100 \mathrm{mV}$ for the $282 \mathrm{~nm}$ beads, but it is not possible to assign a zeta potential to the smallest size except at the highest suspending medium conductivity of $0.1 \mathrm{~S} / \mathrm{m}$.

Values of $K_{\text {Stern }}$ and $\zeta$-potential were also obtained from the best fits to the DEP cross-over measurements, and these are plotted as a function of particle diameter in Fig. 5. Fig. 5a shows that the surface conductance of the particles $K_{\mathrm{S}}$ does not vary widely with particle diameter, but does show a small decrease as the particle size is reduced. In addition to the DEP derived data, Fig. $5 b$ also shows the $\zeta$-potential for particles calculated from the electrophoretic mobility data. The plot shows three separate data sets, one calculated from Eq. (10) using Ohshima's model in the low conductivity limit (open symbols). Also shown in the figure are the results calculated from the model of Shubin, using
Eqs. (13) and (14), with and without the inclusion of the Stern layer. These data were calculated at the limit of the applicability of the model, at $\kappa a=10$. The figure shows that the $\zeta$-potentials, calculated from the DEP measurements using a model which includes both Stern layer and diffuse layer conductance (Eqs. (4) and (5)), are in a good agreement with $\zeta$-potentials calculated from the electrophoretic data using models without Stern layer contribution (both Ohshima and Shubin's models). These calculations show that the $\zeta$-potential is almost invariant with particle size. However, the inclusion of the Stern layer into the model leads to strong particle size dependence.

At low suspending medium conductivities $\left(10^{-4} \mathrm{~S} / \mathrm{m}\right)$, the dielectrophoretic properties of the particle are dominated by surface conductance, which is dominated by the Stern layer $[26,27]$. For the DEP data, the relationship between the Stern and diffuse layer conductances can be illustrated by reference to Fig. 6 . Here both conductances are plotted as a function of suspending medium conductivity for three different particle sizes. The data in this plot was calculated with a value for a fixed $\zeta$-potential, taken from the data summarised in Table 2. Although the particle $\zeta$-potential varies with solution conductivity (cf. Fig. 4), the first approximation of a fixed value enables an order of magnitude comparison to be made between the respective contributions of the Stern and diffuse layer. The figure shows that for larger particles, the diffuse layer contribution is insignificant up to quite high conductivities, of the order of $10^{-1} \mathrm{~S} / \mathrm{m}$. For smaller particles the diffuse layer begins to make a significant contribution at lower suspending medium conductivities, e.g., $10^{-2} \mathrm{~S} / \mathrm{m}$ for the $44 \mathrm{~nm}$ particles. The fact that the surface conductance remains approximately constant with suspending medium conductivity has been noted by other workers, 

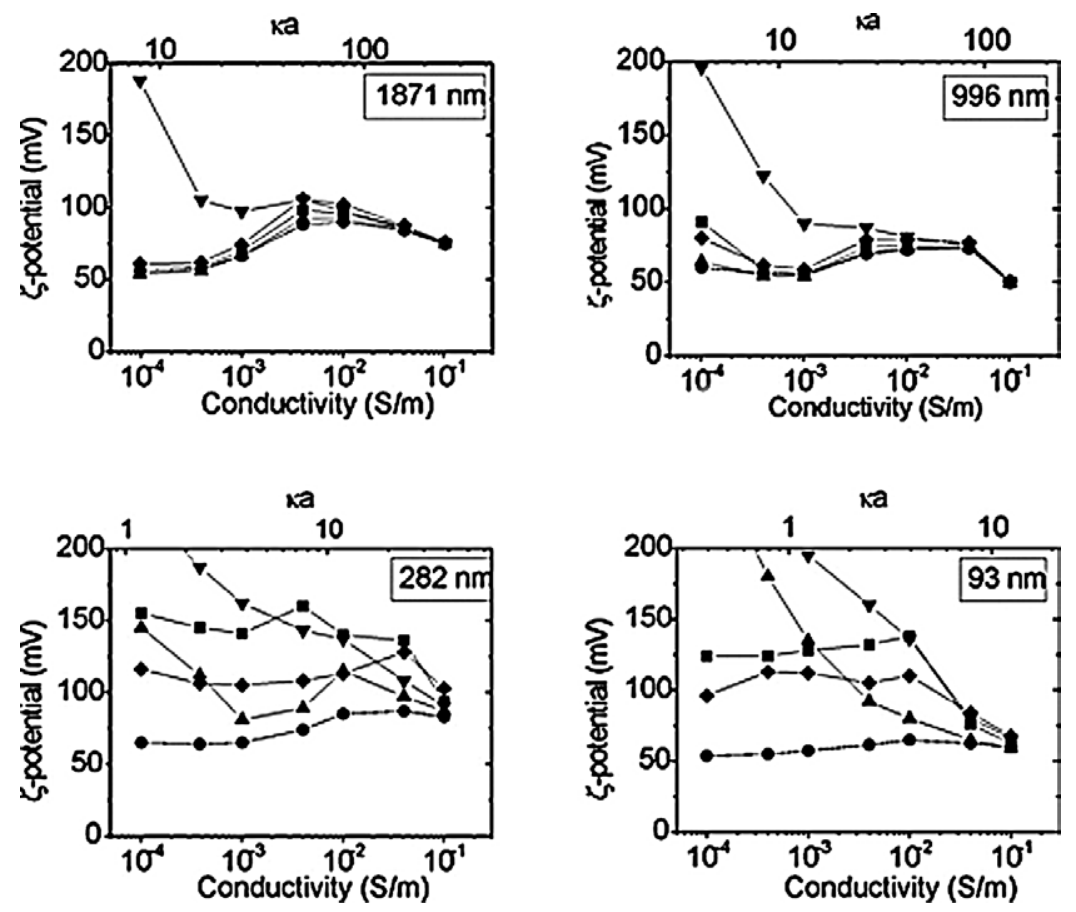

Fig. 4. The $\zeta$-potential of 93, 282, 966 and $1871 \mathrm{~nm}$ diameter beads calculated from the electrophoretic mobility measurements by using different models:

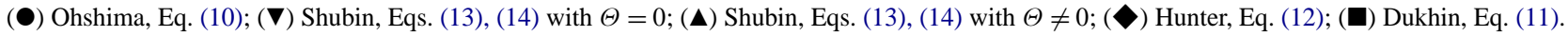

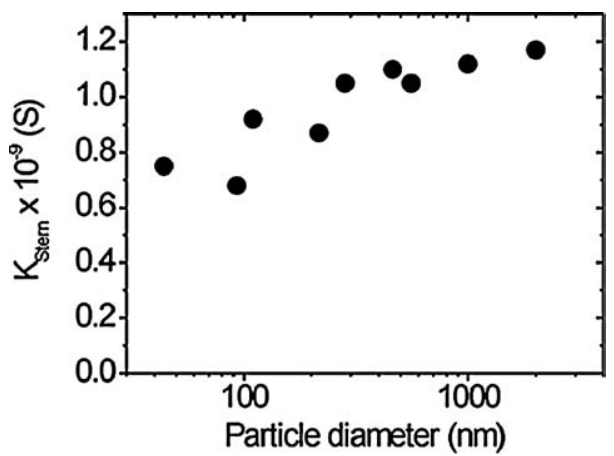

(a)

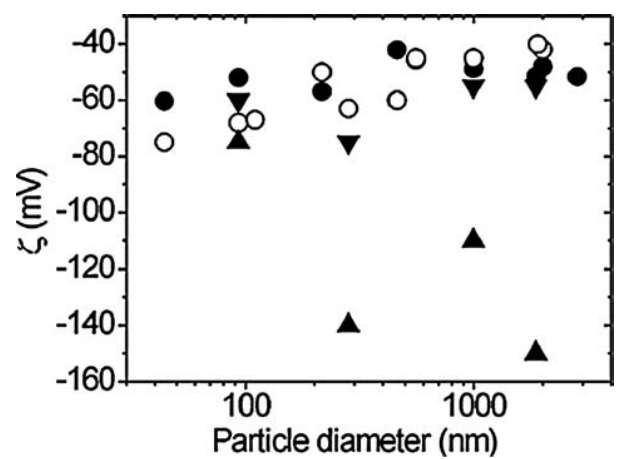

(b)

Fig. 5. (a) The Stern layer conductance $\left(K_{\text {Stern }}\right)$ determined from the DEP cross-over measurements plotted for different particle diameters. (b) The $\zeta$-potential data calculated from the DEP cross-over measurements for different particle diameters $(\bigcirc)$. Also shown in this figure is the $\zeta$-potential data obtained from the electrophoretic mobility using Ohshima's model (Eq. (10)) (@) at the limiting low conductivity value, and Shubin's model (Eqs. (13), (14)) at the conductivity corresponding to $\kappa a=10$ (see Fig. 4), with $(\boldsymbol{\Delta})$ and without $(\boldsymbol{\nabla})$ the Stern layer.

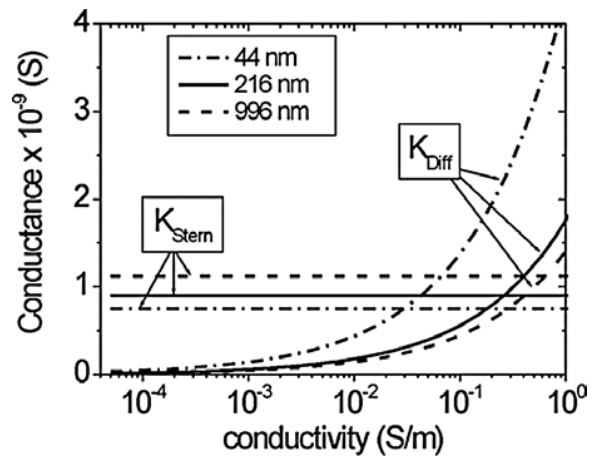

Fig. 6. Conductance of the Stern layer $\left(K_{\text {Stern }}\right)$ and the diffuse part $\left(K_{\text {Diff }}\right)$ of the double layer for the latex particles as a function of the suspending medium conductivity. Calculation was performed using parameters from Table 2 for 44, 216, and $996 \mathrm{~nm}$ beads.

notably Minor et al. [41,42]. They performed plug conductivity measurements together with low frequency dielectric measurements on sulphate latex and obtained a constant surface conductance of $5.7 \mathrm{nS}$ over a wide range of molarities. This result implies that the countercharge does not change with suspending medium molarity, and that the ion mobility in the double layer remains constant with molarity. Both of these points are consistent with the Gouy-Chapman double layer model where the Stern layer charge is unaffected.

The data summarised in Table 2 shows that the surface conductance of the particles as determined from the DEP data is almost independent of particle size and falls within the range 0.75 to $1.2 \mathrm{nS}$, which is typical of other values of surface conductance measured for latex particles by other means. Taking $1 \mathrm{nS}$ as a typical conductance, and set- 
Table 3

Diffuse layer charge density of particles calculated from electrophoretic $\zeta$-potential measurements using three different models, calculated at $\kappa a=10$; also shown is the Stern layer charge density calculated from the DEP surface conductance $(K s)$ at the lower limit of suspending medium conductivity

\begin{tabular}{|c|c|c|c|c|c|c|}
\hline \multirow{3}{*}{$\begin{array}{l}\text { Particle diameter, } \\
2 a(\mathrm{~nm})\end{array}$} & \multirow{3}{*}{$\frac{\text { DEP }}{\frac{\rho_{\mathrm{q}}, \text { Stern }\left(\mu \mathrm{C} / \mathrm{cm}^{2}\right)}{\text { Eq. (5) }}}$} & \multicolumn{5}{|c|}{ Electrophoresis (at $\kappa a=10$ ) } \\
\hline & & \multicolumn{3}{|c|}{$\rho_{\mathrm{q}, \text { Diffuse }}\left(\mu \mathrm{C} / \mathrm{cm}^{2}\right)$} & \multirow[t]{2}{*}{$\Theta$} & \multirow[t]{2}{*}{$\sigma_{\mathrm{m}}(\mathrm{mS} / \mathrm{m})$} \\
\hline & & Eq. (10) & Eqs. (13) and (14), $\Theta=0$ & Eqs. (13) and (14), $\Theta \neq 0$ & & \\
\hline 93 & 0.88 & 1.0 & 1.17 & 1.6 & 0.75 & 65 \\
\hline 282 & 1.36 & 0.43 & 0.78 & 1.88 & 0.49 & 7 \\
\hline 996 & 1.45 & 0.12 & 0.105 & 0.32 & 3.2 & 0.65 \\
\hline 1871 & 1.53 & 0.05 & 0.05 & 0.41 & 2.2 & 0.15 \\
\hline
\end{tabular}

ting the mobility of the ions to their bulk value, this gives a charge density in the Stern layer of $1.3 \mu \mathrm{C} / \mathrm{cm}^{2}$, similar to that measured by Minor et al. [41,42] for sulphate latex at $1.61 \mu \mathrm{C} / \mathrm{cm}^{2}$. Lyklema and Minor [31] calculated the diffuse layer charge density of plugs of $800 \mathrm{~nm}$ diameter sulphated polystyrene. They estimated $\rho_{\mathrm{q}, \text { Diff }}=3.9 \mu \mathrm{C} / \mathrm{cm}^{2}$ and a Stern charge density of $\rho_{\mathrm{q} \text {.Stern }}=2.4 \mu \mathrm{C} / \mathrm{cm}^{2}$. With the Stern layer charge mobility approximately $89 \%$ of the bulk value, they deduced that the ratio $\Theta$ is approximately 0.56 , so that half the surface conduction occurs behind the plane of shear. Midmore et al. [22] came to similar conclusion from electrophoresis data.

However, different ratios of Stern layer to diffuse layer conductance were found by Werner et al. [43] in their measurement of streaming potentials of materials. They investigated plasma deposited fluorpolymer, and showed that the Stern layer conductance dominated the streaming potential data by a factor of 10 over the diffuse layer conductance.

The surface charge density in the diffuse layer can be calculated from the Grahame equation, which for a monovalent symmetric electrolyte and low surface potential $\sim 50 \mathrm{mV}$ is given by

$\rho_{\mathrm{d}}^{2}=8 \varepsilon_{\mathrm{m}} k T n_{0} \sinh \left(\frac{q \zeta}{2 k T}\right)$

For small surface charge densities, this expression can be used to determine the charge density in the diffuse layer from the zeta potential. Table 3 shows the charge density calculated from the electrophoretic mobility data from this equation using the value of zeta potential determined at $\kappa a=10$. Also shown in the table is the Stern layer charge density calculated via Eq. (5), assuming that the Stern layer ions move with their bulk mobility.

The table shows that the charge density in the Stern layer as determined from the DEP measurements is almost independent of particles size, except for the $93 \mathrm{~nm}$ particles, and falls in the range of 0.9 to $1.5 \mu \mathrm{C} / \mathrm{cm}^{2}$. The table also shows that the diffuse layer charge density calculated from the EP mobility, at a given value of $\kappa a$ varies by one order of magnitude from the smallest bead to the largest. The mobile charge seems to be confined entirely to the Stern layer for the larger particle (1871 nm diameter), whereas for the smaller particles the diffuse layer and Stern layer charge density are similar.

\section{Viscosity dependence}

Measurements of the cross-over frequency were made for the particles suspended in $\mathrm{KCl}$ with different viscosities. With reference to Figs. 1 and 2, it can be seen that the cross-over frequency and electrophoretic mobility are almost independent of suspending medium conductivity (or molarity) at the low end of the spectrum, up to a conductivity of approximately $2 \mathrm{mS} / \mathrm{m}$. Therefore the effect of viscosity on the behaviour of the particles was determined by measuring cross-over data at different viscosities with an electrolyte conductivity of less than $1.8 \mathrm{mS} / \mathrm{m}$.

Fig. 7 shows the cross-over frequencies for 110, 216 and $460 \mathrm{~nm}$ diameter beads as a function of suspending medium viscosity (Ficoll concentration). The data clearly indicates that the cross-over frequency decreases with increasing suspending medium viscosity. These results were analysed using three different approaches:

(a) Assume that $K_{\text {Stern }}$ is independent of viscosity and takes values given by Eqs. (4) and (5), summarised in Table 2 . The diffuse layer conductance, $K_{\text {Diff }}$, is set to be approximately inversely proportional to viscosity, through two known parameters, $D$ the diffusion constant of the ions (related to the viscosity of the electrolyte) and $m$ the electroosmotic flux (cf. Eq. (7)). However, because the Stern layer conductance dominates the behaviour of the particles for most of the conductivity range (Fig. 6), this approach does not describe the experimental data.

(b) In the second model the mobility of the ions in the Stern layer is assumed to be related to the bulk viscosity. The data was analysed by setting $K_{\text {Stern }} \propto 1 / \eta$, with $K_{\text {Diff }}$ as for (a) above. The fit using this approach is shown in Fig. 7a, indicating that the cross-over frequency should decrease much quicker than observed experimentally.

(c) The experimental data shows that the cross-over reaches a plateau at high viscosity. This phenomenon was empirically modelled by dividing the Stern layer conductance into two components, one of a constant value and a second which is function of the relative viscosity:

$K_{\text {Stern }, \eta}=\frac{K_{\text {Stern }}}{2}\left(1+\frac{\eta_{0}}{\eta}\right)$.

The ratio $\eta / \eta_{0}$ is a dimensionless parameter reflecting the relative change in suspension viscosity after addition of Fi- 


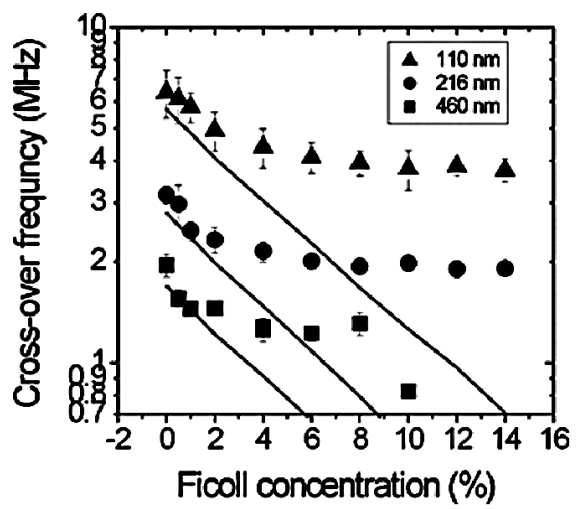

(a)

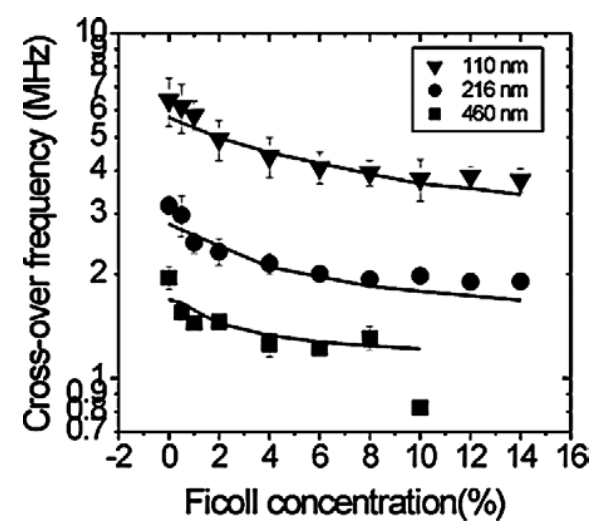

(b)

Fig. 7. DEP cross-over frequency for $110(\boldsymbol{\nabla}), 216(\boldsymbol{O})$ and $460 \mathrm{~nm}$ (ם) diameter latex beads as a function of suspending medium viscosity (Ficoll concentration). The points are experimental data. The two sets of lines shown in (a) and (b) are fits calculated with the following assumptions: (a) $K_{\text {Stern }}$ inversely proportional to viscosity; (b) $K_{\text {Stern, } \eta}=$ $\left(K_{\text {Stern }} / 2\right)\left(1+\eta_{0} / \eta\right)$. In both cases $K_{\text {Diff }}$ is a function of viscosity through the parameters $m$ and $D$ of Eq. (6). The molarity of the suspending medium was calculated from the measured conductivity. Values of $\zeta$-potential and $K_{\text {Stern }}$ at $0 \%$ Ficoll were taken from the low-viscosity limit of the fits to the cross-over data plotted in Fig. 1 and Table 2.

coll to the suspending solution. In this expression, $\eta$ is absolute viscosity of the Ficoll suspension and $\eta_{0}$ the viscosity of pure water. The results of this model are presented in Fig. $7 \mathrm{~b}$ and show good agreement with experiment.

The viscosity cross-over data can also be replotted to take into account the values of the suspending medium conductivity. This is shown in Fig. 8, where cross-over data for different viscosities (cf. Fig. 7) is plotted at the corresponding suspending medium conductivity, for three sizes of particles (open circles). Also shown in the plot for comparison is the data for beads suspended in Ficoll-free $\mathrm{KCl}$ (from Fig. 1), solid points. The two lines represent best fits to the experiment for two limits of viscosity. The dashed line shows the predicted cross-over frequency spectrum calculated for the maximum viscosity $\left(9.2 \times 10^{-3} \mathrm{~kg} /(\mathrm{m} \mathrm{s})=14 \%\right.$ Ficoll). It can be seen from this plot that the cross-over data is insensitive to suspending medium conductivity (for $\sigma<$ $10^{-3} \mathrm{~S} / \mathrm{m}$ ), implying that the cross-over frequency is influ-
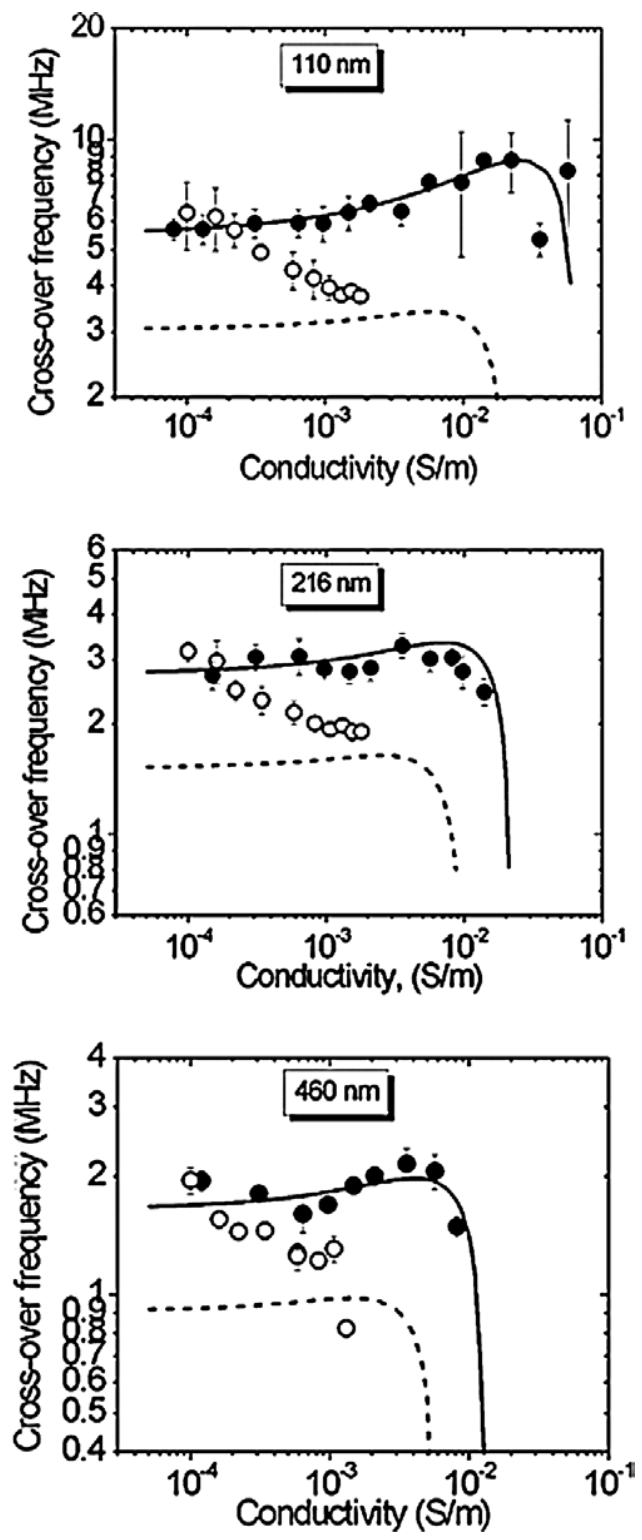

Fig. 8. Summary graph showing the DEP cross-over data of beads at different suspending media viscosity and conductivity. Solid points correspond to cross-over data measured in Ficoll-free $\mathrm{KCl}$. The open circles are data at different Ficoll concentrations plotted at the appropriate conductivity value, as summarised in Table 1. Solid and dashed lines are theoretical predictions for the two limits of viscosity ( $\mathrm{KCl}$ with $0 \%$ and $14 \%$ Ficoll, respectively), obtained from Eqs. (2)-(7).

enced only by the suspending medium viscosity rather than by conductivity in this region.

It is tempting to analyse this last model (c) in the light of the triple model of the interfacial region. This model divides the region into the inner and outer Helmholtz planes, and the diffuse layer. The outer Helmholtz plane (oHp) delineates the beginning of the diffuse layer, but the plane of shear may be coincident with the oHp or lie a small distance away from this position. Shubin et al. [38] estimated this distance to be of the order of 1 to $3 \mathrm{~nm}$, depending on the suspending medium molarity. They considered two models, 
one in which the surface conduction occurs only in the diffuse layer but restricted to the thin region between the outer Helmholtz plane and the beginning of the shear plane. The alternative model identifies the zeta potential with the oHp and all the anomalous surface conductivity is restricted to the Stern layer. This second model was shown to have the most consistent interpretation of their data. A similar distance between the oHp and shear plane of about $1.2 \mathrm{~nm}$ was estimated by Midmore et al. [22] for cationic latex.

The fixed Stern layer conductance (independent of viscosity) may be associated with charge movement in the inner Helmholtz planes (iHp). The second component may be associated with a thin layer of charge inside the slip layer but sufficiently far from the surface to be influenced by the bulk viscosity of the suspending medium. Recently molecular dynamic simulations showed that charge located in the immobile or stagnant Stern layer could in fact move into the diffuse layer and contribute to a surface conductivity with the mobility of the charge apparently equal to that of the bulk [44].

\section{Conclusion}

In this paper we have presented a comprehensive study and comparison of both the $\mathrm{AC}$ and DC electrokinetic properties of submicrometre latex particles. The properties of the particles were measured as functions of size, suspending medium conductivity and suspending medium viscosity. The data was analysed in the framework of different analytical models describing the electrokinetic behaviour of the particles. The electrokinetic data can be explained using a theory that models charge transport as the superposition of the contribution from the Stern and the diffuse layer.

Experimental observations of the DEP behaviour of particles in different viscosity media show that the Stern layer conductance can be described as the sum of two components, of which one is suspending medium-invariant and the other is a function of the relative viscosity of the suspending medium. We postulate that one component may be associated with the inner Helmholtz plane, and the other with the mobile charge behind the shear plane.

\section{Acknowledgments}

This work was supported by Leverhulme Trust Foundation, Grant No. F/00179/D. The authors thank Dr. Nicolas Green for fruitful discussions.

\section{References}

[1] S.S. Dukhin, B.V. Deryaguin, in: E. Matijevic (Ed.), Surface and Colloid Science, vol. 7, Wiley, New York, 1974.

[2] A.E.J. Meijer, W.J. van Megan, J. Lyklema, J. Colloid Interface Sci. 66 (1978) 99.
[3] R.J. Hunter, Zeta Potential in Colloid Science, Academic Press, New York, 1981.

[4] C.N. Bensley, R.J. Hunter, J. Colloid Interface Sci. 92 (1983) 448 462.

[5] C.F. Zukoski, D.A. Saville, J. Colloid Interface Sci. 107 (1985) 322.

[6] W.B. Russel, D.A. Saville, W.R. Schowwalter, Calloidal Dispersions, Cambridge Univ. Press, Cambridge, 1989.

[7] J. Lyklema, Fundamentals of Interface and Colloid Science, Academic Press, London, 1995.

[8] J. Kijlstra, H.P. van Leeuwen, J. Lyklema, J. Chem. Soc. Faraday Trans. 88 (1992) 3441.

[9] M.R. Gittings, D.A. Saville, Langmuir 11 (1995) 798-800.

[10] M. Rasmusson, S. Wall, J. Colloid Interface Sci. 209 (1999) 312-326.

[11] J. Lyklema, H.P. van Leeuwen, M. Minor, Adv. Colloid Interface Sci. 83 (1999) 33-69.

[12] R.W. O'Brien, L.R. White, J. Chem. Soc. Faraday Trans. 74 (1978) 1607.

[13] D.F. Myers, D.A. Saville, J. Colloid Interface Sci. 131 (1989) 461.

[14] D.E. Dunstan, L.R. White, J. Colloid Interface Sci. 152 (1992) 308.

[15] R. Barchini, D.A. Saville, J. Colloid Interface Sci. 173 (1995) 86-91.

[16] M. Rasmusson, B.E. Mellander, J. Ennis, J. Colloid Interface Sci. 209 (1999) 327-340.

[17] L. Vorwerg, M. Antonietti, K. Tauer, Colloids Surf. A 150 (1999) 129 135.

[18] F. Carrique, L. Zurita, A.V. Delgado, Colloids Surf. 92 (1994) 9.

[19] C. Chassagne, D. Bedeaux, J.P.M. van der Ploeg, G.J.M. Koper, Langmuir 19 (2003) 3619-3627.

[20] R.J. Hunter, Adv. Colloid Interface Sci. 100 (2003) 153-167.

[21] J.J. Bikerman, Z. Phys. Chem. A 163 (1933) 378.

[22] B.R. Midmore, D. Diggins, R.J. Hunter, J. Colloid Interface Sci. 129 (1989) 153-161.

[23] J.J. Bikerman, J. Chim. Phys. 32 (1935) 285.

[24] J.B. Miles, H.P. Robertson, Phys. Rev. 40 (1932) 583.

[25] C.T. O'Konski, J. Phys. Chem. 64 (1960) 605-619.

[26] N.G. Green, H. Morgan, J. Phys. Chem. 103 (1999) 41.

[27] M.P. Hughes, H. Morgan, M.F. Flynn, J. Colloid Interface Sci. 220 (1999) 454-457.

[28] W.M. Arnold, H.P. Schwan, U. Zimmermann, J. Phys. Chem. 91 (1987) 5093

[29] M.P. Hughes, H. Morgan, Anal. Chem. 71 (1999) 3441-3445.

[30] M.P. Hughes, N.G. Green, J. Colloid Interface Sci. 250 (2002) 266268.

[31] J. Lyklema, M. Minor, Colloids Surf. A 140 (1998) 33-41.

[32] H.A. Pohl, Dielectrophoresis, Cambridge Univ. Press, Cambridge, 1978.

[33] T.B. Jones, Electromechanics of Particles, Cambridge Univ. Press, Cambridge, 1995.

[34] H. Morgan, N.G. Green, AC Electrokinetics: Colloids and Nanoparticles, RSP, Herts, UK, 2003.

[35] A. Ramos, H. Morgan, N.G. Green, A. Castellanos, J. Phys. D 31 (1998) 2338-2353.

[36] H. Ohshima, J. Colloid Interface Sci. 239 (2001) 587-590.

[37] R.W. O’Brien, R.J. Hunter, Can. J. Chem. 59 (1981) 1878-1887.

[38] V.E. Shubin, R.J. Hunter, R.W. O’Brien, J. Colloid Interface Sci. 159 (1993) 174-183.

[39] X. Chen, H.C. Berg, Biophys. J. 78 (2000) 1036-1041.

[40] B.D. Midmore, G.V. Pratt, T.M. Herrington, J. Colloid Interface Sci. 184 (1996) 170-174.

[41] M. Minor, A.J. van der Linde, J. Lyklema, J. Colloid Interface Sci. 203 (1998) 177-188.

[42] M. Minor, H.P. van Leeuwen, J. Lyklema, J. Colloid Interface Sci. 206 (1998) 397-406.

[43] C. Werner, R. Zimmermann, T. Kratzmuller, Colloids Surf. A 192 (2001) 205-213.

[44] J. Lyklema, S. Rovillard, J. De Coninck, Langmuir 14 (1998) 5659 5663. 